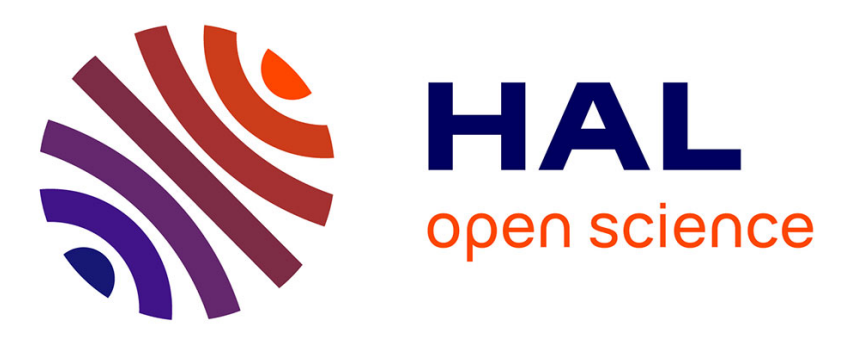

\title{
On the dynamic fragmentation of glass: a meso-damage model
}

\author{
Xavier Brajer, François Hild, Stéphane Roux
}

\section{To cite this version:}

Xavier Brajer, François Hild, Stéphane Roux. On the dynamic fragmentation of glass: a meso-damage model. International Journal of Fracture, 2010, 163, pp.121-131. 10.1007/s10704-009-9421-9 . hal00430487

\section{HAL Id: hal-00430487 \\ https://hal.science/hal-00430487}

Submitted on 7 Nov 2009

HAL is a multi-disciplinary open access archive for the deposit and dissemination of scientific research documents, whether they are published or not. The documents may come from teaching and research institutions in France or abroad, or from public or private research centers.
L'archive ouverte pluridisciplinaire HAL, est destinée au dépôt et à la diffusion de documents scientifiques de niveau recherche, publiés ou non, émanant des établissements d'enseignement et de recherche français ou étrangers, des laboratoires publics ou privés. 
Int. J. Fract. manuscript No.

(will be inserted by the editor)

ON THE DYNAMIC FRAGMENTATION OF GLASS:

A MESO-DAMAGE MODEL

Xavier Brajer • François Hild .

Stéphane Roux

Received: date / Accepted: date

\begin{abstract}
This paper presents an anisotropic damage model to deal with the fragmentation induced by impact loadings on glass samples. As small-scale (i.e., sub-element) damage is described as well as cracks extending above the element scale, an approach referred to as "meso-damage" is developed. The latter, which is based on the knowledge of random distributions of initiation sites, predicts different regimes such as single or multiple fragmentation. The experimental opening crack pattern obtained in edge-onimpact test is reproduced numerically.
\end{abstract}

X. Brajer

Saint-Gobain Recherche

39 Quai L. Lefranc, F-93303 Aubervilliers Cedex, France

E-mail: xavier.brajer@saint-gobain.com

F. Hild, S. Roux

LMT-Cachan, ENS Cachan / CNRS / UPMC / PRES UniverSud Paris

61 avenue du Président Wilson, F-94235 Cachan Cedex, France

E-mail: \{francois.hild,stephane.roux\}@lmt.ens-cachan.fr 
Keywords FE simulations · fragmentation regime $\cdot$ non-local damage Poisson-

Weibull model

\section{Introduction}

Impact resistant materials are being developed for armor applications $[1,2]$. For example, glass is used against ballistic threats (e.g., in windshields). For the latter application, multi-layered armor materials are used (with polycarbonate, PC, as back layer and polyurethane, $\mathrm{PU}$, between glass plies) to optimize the ballistic performance. The use of PU and PC is essential for maintaining the fragments when glass is impacted, especially for the back layer that ensures the structural integrity and absorbs part of the impact energy. The work presented herein aims at modeling the fragmentation of sodalime silicate glass impacted by a bullet of .44 Magnum type. Edge-on-impact (EOI) configurations [3-6] allow for the visualization of cracking during the experiment by using a high-speed camera, or thereafter when a sarcophagus configuration is utilized [7]. Different cracking patterns are observed. The present work intends to describe numerically the cracking features observed experimentally.

To predict crack patterns in brittle materials dynamic loading conditions, several approaches are followed [8]. Some computational modeling directly uses discrete descriptions of fracture [9-12], or models based upon Smoothed Particle Hydrodynamics $[13,14]$ to simulate the behavior of brittle materials under shock loading [15]. On the other side of the modeling spectrum, continuum models have been used for glass (e.g., Ref. [16]) but the relationship with the microstructure is remote, and the description of damage is implicit. To account for multiple microcracking under dynamic loading conditions, the early models are based upon the analysis of cracked systems $[17,18]$ on a 
continuum level at which constitutive equations are written. The models developed by Taylor et al. [19] and Rajendran [20] use the previous framework. This approach usually leads to an isotropic description of damage. Cracking is essentially anisotropic and the model needs to account for it to be realistic in terms of actual damage predictions [21, 22]. Combining discrete features and continuum descriptions are also possible [23, 22]. The latter approach will be followed herein.

Based upon a dynamic fragmentation analysis, an anisotropic damage model is introduced. The latter uses characteristic parameters that depend on material properties (i.e., distributions of surface and bulk initiation sites in glass [5]) and loading conditions. 3D-FE simulations are performed and analyzed with respect to experimental observations. Within a finite element modeling framework, a procedure is proposed to describe on the same footing micro-cracks (responsible for local damage) and mesocrack propagation. The mesh size that allows for this distinction has to be made transparent to this multiscale description. We will use the term "meso-damage" to refer to this modeling. These developments are implemented in an explicit finite element code to model edge-on-impacts. The transition between multiple fragmentation close to the impact zone and single fragmentation far from impact is studied with the meso-damage version of the model.

\section{Experimental results}

Bullets are fired by a gun and impact a glass target of surface area $100 \times 100 \mathrm{~mm}^{2}$ and of thicknesses varying between 8 and $15 \mathrm{~mm}$. The projectile speed is measured by two optical cells one meter apart. An "open" configuration is used enabling for in-situ observations by utilizing a high-speed camera (Ultranac FS 501, time of exposure: $1 \mu \mathrm{s}$ ). 
When the bullet reaches the second cell, flashlights are triggered. The bullet impact on the target triggers the camera to take pictures. According to the European standard EN1063 [24], one of the threats for armors is a "soft" bullet (e.g., .44 magnum) further referred to as "BR4," traveling at a (moderate) speed of $430 \mathrm{~m} / \mathrm{s}$. BR4 bullets have a soft core made of lead and a brass envelope (Figure 1).

Typical results obtained by using the high-speed camera are shown in Figure 1. When the bullet impacts the glass sample and induces cracking a few microseconds thereafter, dark zones appear on the pictures. They correspond to damaged zones where glass is no longer transparent (caused by the light scattering by crack surfaces). One observes the damage zone propagating in the sample and the crack front is faithfully identified by this type of inspection and consequently propagation velocities are determined with a good accuracy. After impact, fragments of the impacted sample are scattered and the BR4 core (made of lead) is fully sublimated.

A "sarcophagus" configuration that maintains fragments together on a glass target of size $100 \times 100 \times 10 \mathrm{~mm}^{3}$ is also used (Figure 2). After impact, a hyper-fluid (EPOFIX) resin infiltrates (in vacuum for $12 \mathrm{~h}$ ) the cracks and maintains the fragments together to observe the impacted sample. The projectile does not penetrate the target. There are different zones that characterize the interaction between the projectile and the target. A first zone in the vicinity of the impact is totally comminuted. A second area displays a high density of radial and hoop cracks. A third zone appears in which only few long cracks propagate. These zones correspond to different cracking features in brittle materials: 
- multiple fragmentation in the first zone with mainly closed cracks (glass is totally comminuted; the size of thinner fragments is estimated to be a few micrometers), this fragmentation is caused by high shear stresses just under the contact zone;

- multiple fragmentation under mode I loading in the second zone, due to high tensile stresses induced by the radial motion of the initial compressive wave;

- single fragmentation in the third zone (consisting mainly of radial macrocracks).

\section{Fragmentation analysis}

When a bullet impacts a glass target, a compressive stress wave propagates ahead of the impacted area, with the speed of longitudinal acoustic waves [25]. The radial motion also induces tensile stresses that nucleate microcracks. It is assumed that the population of initiation sites leading to damage and failure in glass is identical when the material is subjected to quasi-static and dynamic loading conditions.

\subsection{Poisson point process}

The material is assumed to contain initiation points with random critical stresses $\sigma_{c r}$. For each applied stress level $\sigma$, points whose critical stress is less than $\sigma$ are potential initiation sites. Their density is denoted by $\lambda_{i n}$ (or equivalently, the statistical distribution of initiation sites follows a Poisson point process of intensity $\left.\lambda_{\text {in }}[26-29]\right)$. Consequently, the probability of finding $\delta$ points within a uniformly loaded domain $\Omega$ of (surface or volume) measure $Z$ reads

$$
P(\delta, \Omega)=\frac{\left(\Lambda_{i n}\right)^{\delta}}{\delta !} \exp \left(-\Lambda_{i n}\right)
$$

so that $\Lambda_{\text {in }}$ corresponds to the average number of points within $\Omega$. By definition, $\Lambda_{\text {in }}$ is related to the density $\lambda_{i n}$ by $\Lambda_{i n}=\lambda_{i n} Z$ for a uniformly loaded domain. The larger 
the applied stress $\sigma$, the greater the number of points for which $\sigma_{c r}<\sigma$. One possible choice to account for this trend is given by a power law function of the applied stress $\sigma$

$$
\lambda_{i n}(\sigma)=\lambda_{0}\left(\frac{\langle\sigma\rangle}{\sigma_{0}}\right)^{m}
$$

where $m$ and $\sigma_{0}^{m} / \lambda_{0}$ will be interpreted as the Weibull parameters when single fragmentation occurs, and $\langle\bullet\rangle$ denotes the Macauley brackets indicating that initiation is governed by tensile stresses.

\subsection{Single fragmentation}

In a $3 \mathrm{D}$ setting, the previous analysis holds for a stress $\sigma$ corresponding to maximum principal stress $\sigma_{1}$. Under quasi-static loading condition, a weakest link hypothesis usually holds [30]. Therefore, the failure probability $P_{F}$ is the probability of finding at least one initiation site within $\Omega$ when $\sigma_{1} \equiv \sigma_{F}>0$

$$
P_{F}=P(\delta \geq 1, \Omega)=1-P(\delta=0, \Omega)=1-\exp \left[-\lambda_{0} Z\left(\frac{\sigma_{F}}{\sigma_{0}}\right)^{m}\right]
$$

when a uniform stress is applied. If the stress field is heterogeneous, $\Lambda_{\text {in }}$ is related to $\lambda_{\text {in }}$ by

$$
\Lambda_{i n}\left(\sigma_{F}\right)=\int_{\Omega} \lambda_{i n}\left[\sigma_{1}(\mathbf{x})\right] \mathrm{d} \mathbf{x}
$$

and the failure probability is written as

$$
P_{F}=1-\exp \left[-\lambda_{0} Z_{\text {eff }}\left(\frac{\sigma_{F}}{\sigma_{0}}\right)^{m}\right]
$$

where $\sigma_{F}$ denotes the failure stress

$$
\sigma_{F}=\max _{\Omega} \sigma_{1}(\mathbf{x})
$$

and $Z_{\text {eff }}$ the effective volume or surface [31]

$$
Z_{\mathrm{eff}}=\int_{\Omega}\left[\frac{\left\langle\sigma_{1}(\mathbf{x})\right\rangle}{\sigma_{F}}\right]^{m} d \mathbf{x}
$$


associated with the maximum principal stress $\sigma_{1}$ when positive. The mean failure stress $\bar{\sigma}_{F}$ is then expressed as

$$
\bar{\sigma}_{F}=\sigma_{0}\left(\lambda_{0} Z_{\text {eff }}\right)^{-\frac{1}{m}} \Gamma\left(1+\frac{1}{m}\right)
$$

and describes the fact that the larger the volume (or surface), the smaller the mean failure stress. $\Gamma(a)$ denotes the (Euler) Gamma function

$$
\Gamma(a)=\int_{0}^{+\infty} t^{a-1} \exp (-t) d t
$$

The microstructure model accounts for random distributions of initiation sites. The Poisson-Weibull model enables one to relate the Weibull parameters to microstructural properties describing the population of initiation sites. The latter is the key for understanding probabilistic features related to the fragmentation of brittle materials. In this first case, the initiation conditions are the only ones that control the failure properties. The Weibull parameters used for glass are either surface [32] or bulk [33] parameters. Both will be considered in the sequel.

\subsection{Multiple fragmentation}

Under dynamic loading conditions, a propagating crack may shield other initiation sites because of the dynamic growth of a domain of measure $Z_{o}$ where stresses are relaxed. The cracking pattern then results from a competition between obscuration (or shielding) phenomena and the loading rate (Figure 3) initiating new cracks. The density of initiation sites $\lambda_{i n}$ is divided into two parts, namely, $\lambda_{b}$ the crack density, and $\lambda_{o}$ the density of obscured sites. The obscuration probability, which will be used to relate these three densities, is expressed as an extension of the classical Weibull 
law [34]

$$
P_{o}=1-\exp \left[-\hat{Z}_{o} \lambda_{i n}(\sigma)\right] \quad \text { with } \quad \hat{Z}_{o} \lambda_{i n}(\sigma) \equiv \int_{0}^{T} Z_{o}(T-t) \frac{\mathrm{d} \lambda_{i n}}{\mathrm{~d} t} \mathrm{~d} t=Z_{o} \star \dot{\lambda}_{i n}
$$

where $\hat{Z}_{o}$ is the measure of the mean obscuration zone, $\star$ the convolution product, corresponds to the first order time derivative, and $Z_{o}$ the size of the obscuration zone. The latter is dependent on the crack propagation velocity $k C_{0}$, a shape factor $S$ and the space dimension $n$

$$
Z_{o}(t)=S\left(k C_{0} t\right)^{n} H(t)
$$

where $H$ is the Heaviside step function. If the loading rate is small enough, $\hat{Z}_{o}$ becomes equal to the entire representative volume or surface, and yields the Weibull failure probability (5). For example, when $n=3$, the growth law of the obscuration probability is derived from Equation (10)

$$
-\frac{\mathrm{d}^{3}}{\mathrm{~d} t^{3}} \ln \left(1-P_{o}\right)=\dddot{Z}_{o} \star \lambda_{i n}=6 S\left(k C_{0}\right)^{3} \lambda_{i n}
$$

Let us assume that the microscopic stress rate $\dot{\sigma}$ is constant. By noting that, in pure tension, the mesoscopic stress $\Sigma$ is related to the microscopic (or effective) stress $\sigma$ by $\sigma=\Sigma /(1-D)[35]$, the ultimate tensile strength $(\mathrm{d} \Sigma / \mathrm{d} \sigma=0)$, denoted by $\Sigma_{\max }$, is then expressed as

$$
\frac{\Sigma_{\max }}{\sigma_{\mathrm{c}}}=\left[\frac{1}{\mathrm{e}} \frac{\Gamma(m+n)}{n ! \Gamma(m+1)}\right]^{\frac{1}{m+n}}
$$

where $\sigma_{c}$ is the characteristic stress associated with dynamic fragmentation

$$
\sigma_{c}=\left[\frac{\sigma_{0}^{m} \dot{\sigma}^{n}}{\lambda_{0} S(k C)^{n}}\right]^{\frac{1}{m+n}}
$$

The normalized ultimate strength only depends upon the Weibull modulus $m$, and the space dimension $n$. The ultimate strength itself is then proportional to $\dot{\sigma}^{n /(m+n)}$. This result is in agreement with experimental data of oil shale [36], microconcrete [37], ceramics and glass [38]. 
It is worth noting that in the present case, not only are the Weibull parameters needed, but also a crack propagation velocity, in addition to the considered stress rate. The multiple fragmentation regime is the result of a competition between new initiations and crack propagations, both of them described by the obscuration probability.

\subsection{Transition}

Equations (8) and (13) define two different regimes. The first one is obtained when a weakest link hypothesis is made. It corresponds to single fragmentation. The second one assumes multiple fragmentation. The transition between "quasi-static" and "dynamic" strength is estimated by the intersection between the weakest link and the multiple fragmentation solutions (Figure 4)

$$
\bar{\sigma}_{F}=\Sigma_{\max }(\dot{\sigma})
$$

A threshold stress rate $\dot{\sigma}_{s m}$ allows for the description of the transition between single and multiple fragmentation regimes

$$
\dot{\sigma} \begin{cases}<\dot{\sigma}_{s m} & \text { single fragmentation } \\ \geq \dot{\sigma}_{s m} & \text { multiple fragmentation }\end{cases}
$$

with

$$
\dot{\sigma}_{s m}=\sigma_{0} k C_{0}\left(\lambda_{0} S\right)^{\frac{1}{n}}\left(Z_{\mathrm{eff}} \lambda_{0}\right)^{\frac{m+n}{m n}}\left[\frac{\mathrm{e} \Gamma(m+1) n !}{\Gamma(m+n)} \Gamma^{m+n}\left(\frac{m+1}{m}\right)\right]^{\frac{1}{n}}
$$

This transition does not only depend on material parameters, but also involves the measure $Z_{\text {eff }}$ of the considered examination domain $\Omega_{e}$. The response of a large structure is "dynamic" for low stress rates even if the material follows a weakest link hypothesis for the same loading applied on a smaller domain. 
These two regimes are depicted in Figure 4 when the surface is endowed with Weibull parameters $m_{s}=7, \sigma_{0 s}=100 \mathrm{MPa}, 1 / \lambda_{0 s}=100 \mathrm{~cm}^{2}$ [32], whereas the bulk is characterized by $m_{v}=30, \sigma_{0 v}=3 \mathrm{GPa}, 1 / \lambda_{0 v}=10^{-6} \mathrm{~mm}^{3}$ [33]. For low strain rates, damage and fracture are dominated by surface initiations (i.e., the ultimate strength corresponding to surface initiations is less than that for initiations in the bulk). Conversely, for very high strain rates (i.e., greater than $10^{9} \mathrm{~s}^{-1}$ ), initiations in the bulk may dominate the fragmentation regime. This result is consistent with experimental observations of glass samples $[39,5]$.

The framework proposed herein is thus valid for describing in a unified way both fragmentation regimes. One of its advantages then lies in the fact that quasi-static experiments may be used to determine the material (Weibull) parameters. Once they are known, they can be used in a situation in which single fragmentation no longer occurs (e.g., dynamic fragmentation), provided the crack propagation velocity is known.

\section{Microcracking analysis}

The previous fragmentation results are now incorporated in an anisotropic damage model [29]. The level of stress and stress rate determine the type of fragmentation, namely single or multiple fragmentation. A so-called multi-scale model [22] is used to determine the transition between single and multiple fragmentation regimes. This fragmentation model accounts for quasi-static and dynamic loading conditions in each examination volume or surface. The formulation chosen here would strictly require that the principal stress directions remain fixed. In practice it is believed that this requirement is not much limiting. 
4.1 Anisotropic damage model

Up to now, only the maximum principal stress direction was considered. In the following, anisotropy has to be implemented to account for the directionality of cracking as observed experimentally. By averaging over an examination domain, the mesoscopic principal stress $\Sigma_{i}(i=1, n)$ is equal to $\sigma_{i}\left(1-D_{i}\right)$, where $\sigma_{i}$ is the local (i.e., microscopic and unobscured) principal stress. The damage variable $D_{i}$ is equal to $P_{o}$ associated with a given direction of the eigen stress $i$ (this definition follows the usual assumption of $D_{i}=0$ for an undamaged domain and $D_{i}=1$ for a fully damaged, or obscured, domain [40]), and the growth law of each damage variable $D_{i}$ for bulk initiations is derived from Equation (12)

$$
\frac{\mathrm{d}^{2}}{\mathrm{~d} t^{2}}\left(\frac{1}{1-D_{i}} \frac{\mathrm{d} D_{i}}{\mathrm{~d} t}\right)=6 S\left(k C_{0}\right)^{3} \hat{\lambda}_{i n}\left(\sigma_{i}\right)
$$

when $\sigma_{i}>0$ and $\mathrm{d} \sigma_{i} / \mathrm{d} t>0$, and with the modified density associated with the Weibull model expressed as $[22]$

$$
V_{\mathrm{FE}} \hat{\lambda}_{i n}\left(\sigma_{i}\right)=\left\{\begin{array}{cr}
0 & \text { if } \sigma_{i}<\sigma_{F} \\
\max \left[V_{\mathrm{FE}} \lambda_{0 v}\left(\frac{\sigma_{i}}{\sigma_{0 v}}\right)^{m_{v}}, 1\right] & \text { otherwise }
\end{array}\right.
$$

where $V_{\mathrm{FE}}$ is the volume of the considered finite element (i.e., coincident with the examination domain $\Omega_{e}$ of the fragmentation analysis of Section 3.3). The value $\sigma_{F}$ is a stress obtained by random selection of a real number $R$ lying between 0 and 1 , and inverting $P_{F}\left(\sigma_{F}\right)=R$ when $V_{\mathrm{eff}} \equiv V_{\mathrm{FE}}$ [see Equation (5)]. For surface initiations, only two "in-plane" damage parameters are needed (i.e., one principal stress is always equal to 0) and their growth law read

$$
\frac{\mathrm{d}}{\mathrm{d} t}\left(\frac{1}{1-D_{i}} \frac{\mathrm{d} D_{i}}{\mathrm{~d} t}\right)=2 S\left(k C_{0}\right)^{2} \hat{\lambda}_{i n}\left(\sigma_{i}\right)
$$


when $\sigma_{i}>0$ and $\mathrm{d} \sigma_{i} / \mathrm{d} t>0$, and

$$
S_{\mathrm{FE}} \hat{\lambda}_{i n}\left(\sigma_{i}\right)=\left\{\begin{array}{cc}
0 & \text { if } \sigma_{i}<\sigma_{F} \\
\max \left[S_{\mathrm{FE}} \lambda_{0 s}\left(\frac{\sigma_{i}}{\sigma_{0 s}}\right)^{m_{s}}, 1\right] & \text { otherwise }
\end{array}\right.
$$

where $S_{\mathrm{FE}}$ is the external surface of the considered finite element. Equations (19) and (21) express the average number of initiation sites within a volume or on the surface that is equal to 0, 1 or greater than 1. The integration of Equations (18) and (20) usually assumes zero initial conditions.

The compliance tensor $\mathcal{S}$ is written in the principal frame as [22]

$$
\mathcal{S}=\frac{1}{E}\left[\begin{array}{cccccc}
\frac{1}{1-D_{1}} & -\nu & -\nu & 0 & 0 & 0 \\
-\nu & \frac{1}{1-D_{2}} & -\nu & 0 & 0 & 0 \\
-\nu & -\nu & \frac{1}{1-D_{3}} & 0 & 0 & 0 \\
0 & 0 & 0 & \frac{1+\nu}{\left(1-D_{2}\right)^{\alpha}\left(1-D_{3}\right)^{\alpha}} & 0 & 0 \\
0 & 0 & 0 & 0 & \frac{1+\nu}{\left(1-D_{3}\right)^{\alpha}\left(1-D_{1}\right)^{\alpha}} & 0 \\
0 & 0 & 0 & 0 & 0 & \frac{1+\nu}{\left(1-D_{1}\right)^{\alpha}\left(1-D_{2}\right)^{\alpha}}
\end{array}\right]
$$

where $\alpha=S_{t a} / S_{n o} \approx 1 / 2$, with $S_{t a}$ and $S_{n o}$ are shape factors that describe the obscuration zone of tangential and normal stresses, $E$ Young's modulus of the undamaged material and $\nu$ the corresponding Poisson's ratio.

\subsection{Simulation of EOI experiments}

The edge-on-impact experiment is now simulated by using an explicit finite element code. Materials of the bullets are assumed to behave elasto-plastically. Glass is assumed to be elasto-plastic $(E=70 \mathrm{GPa}, \nu=0.22)$ with a Hugoniot elastic limit (HEL) evaluated at $6 \mathrm{GPa}$. Fragmentation will be described by the previous damage model. The 
FE mesh accounts for surface properties (when needed) endowed with Weibull parameters $m_{s}=7, \sigma_{0 s}=100 \mathrm{MPa}, 1 / \lambda_{0 s}=100 \mathrm{~cm}^{2}$, whereas the bulk is characterized by $m_{v}=30, \sigma_{0 v}=3 \mathrm{GPa}, 1 / \lambda_{0 v}=10^{-6} \mathrm{~mm}^{3}$.

Damage is mainly nucleated on external surfaces of the samples since the maximum strain rates are significantly less than the value $10^{9} \mathrm{~s}^{-1}$, which corresponds to the transition from multiple fragmentation induced by surface and bulk initiations (Figure 4). Figure 5 shows that multiple fragmentation appears in the first half of the target and along the side. This 3D computation is expected to describe accurately the initial stage of bullet impact. It does not account for the propagation of long cracks but only for crack initiation as illustrated in Figure 5. This limitation will be overcome by the introduction of the following "meso-damage" approach where crack extension will be allowed to extend beyond single finite elements.

\section{A "meso-damage" approach}

The present section aims at extending the previous damage model to account for the propagation of long cracks.

\subsection{Extension of the damage model}

New damage parameters $\Delta_{i}$ are introduced in order to describe cracks that are longer than the elements so that the mesoscopic principal stress $\Sigma_{i}$ is now equal to $\sigma_{i}(1-$ $\left.D_{i}\right)\left(1-\Delta_{i}\right)$. Fracture propagation is described by the obscuration zone that grows at a constant speed $k C_{0}=1500 \mathrm{~m} / \mathrm{s}$ [5] from element to element. Again, the damage variables $\Delta_{i}$ are equal to the ratio between the obscuration volume and the total volume in one element (Figure 6) associated with the three cracking directions. The growth of 
$\Delta_{i}$ is obtained by direct derivation of $S\left(k C_{0} t\right)^{3} / V_{\mathrm{FE}}($ when $n=3)$

$$
\frac{\mathrm{d}^{2}}{\mathrm{~d} t^{2}}\left(\frac{\mathrm{d} \Delta_{i}}{\mathrm{~d} t}\right)=6 S\left(k C_{0}\right)^{3} \tilde{\lambda}_{i n}\left(\sigma_{i}\right)
$$

where $\tilde{\lambda}_{i n}$ is equal to $1 / V_{\mathrm{FE}}$, which means that a single mesocrack propagates in the considered element. One may note that this growth law is identical to that used in the case of multiple fragmentation (18) except for the factor $1 /\left(1-D_{i}\right)$ that accounts for overlappings of obscuration zones. All elements where $\Delta_{i} \neq 0$ are listed and they numerically correspond to a crack whose length is that of all elements traversed by the latter. In practice, the local damage variables start to grow, i.e., $D_{i}>0$ (Figure 6a). When $D_{i}$ reaches a critical value $D_{c}$ corresponding to coalescence in the considered element, $\Delta_{i}$ starts to grow towards the neighboring element (Figure $6 \mathrm{~b}$ ). The size of the crack is followed by computing the size of the obscuration zone. Two cases may occur:

- the stress intensity factor is high enough to let it propagate $\left(i . e ., \sigma_{i}\left(1-D_{i}\right) \sqrt{a}>\right.$ $K_{c}$, where $K_{c}$ denotes the toughness of the material) and the element begins to crack and the kinetics of $\Delta_{i}$ is given by Equation (23);

- the stresses are not high enough; no propagation occurs in the neighborhood of the initially damaged element. For the sake of simplicity, the propagation and arrest conditions are associated with the same value of $K_{c}$.

This process is repeated as the stress wave invades the material. The macrocrack propagation may therefore be the result of a series of "microscopic" coalescences when the damage variables $D_{i}$ are high enough $\left(D_{i}>D_{c}\right)$. These multiple events induce a velocity of the crack front that can be greater than $k C_{0}$ and a multiple fragmentation regime is observed. Conversely, the propagation conditions may be satisfied (i.e., 
$\left.\sigma_{i}\left(1-D_{i}\right) \sqrt{a}>K_{c}\right)$ even though the local values of $D_{i}$ are small. A single fragmentation regime is then observed.

\subsection{Effect of element size}

An in-house FE code is used to simulate the propagation of a cylindrical divergent wave [21]. Two element sizes are used when a cavity in a slab is subjected to a pressure step of $-6 \mathrm{GPa}$ (Figure 7 ). The space-time diagram of the damage variable $\Delta_{1}$ is shown when using the meso-damage model. The white zone corresponds to values of $\Delta_{1}$ greater than $D_{c}=0.85$. Two different mesh densities are used and the spacetime diagrams are virtually identical. Except for the scatter induced by the multi-scale damage model, the results are quasi mesh-independent. The damage variable grows up to a given point where it stops when the propagation condition is no longer satisfied.

\subsection{Divergent cylindrical wave}

The previous 3D simulations (Section 4.2) help us in determining a contact force between the .44 Magnum bullet and the surface of the target (Figure 8). During the first microseconds after impact, the initial shock wave propagates followed by the collapse of the projectile so that the contact force increases. For the sake of simplicity, the propagation of a cylindrical divergent wave is assumed.

Figure 9 shows the results obtained by using the damage variable $\Delta_{1}$. In the first part of the interaction, the stress level induced by the compressive wave are high enough to initiate several cracks in each finite element and the damage front velocity is related to the speed at which the induced tensile (hoop) stress develops. The zigzagged profile corresponds to multiple initiations and propagations. The apparent propagation 
velocity of the damage front is greater than $k C_{0}$ since this phenomenon is dominated by initiation events. Later, as observed (Figure 1), long cracks begin to propagate at a constant speed $k C_{0}=1500 \mathrm{~m} / \mathrm{s}$ with no further initiation. In that regime, it is described by propagation conditions. This transition could not have been assessed by using a microscopic damage model alone. Last, the dashed line in Figure 9 shows the result for the experimental location of the crack front evaluated from Figure 1. The two regimes are found and the transition is well captured.

5.4 Edge-on impact

Figure 10 compares the simulations of the edge-on-impact test obtained with the microand mesoscopic versions of the damage model. Multiple fragmentation in the first half of the target and along the side is followed by single fragmentation as already shown in Figure 9. This 3D computation describes more accurately the initial stage of bullet impact and the propagation of long cracks (Figure 1) than with the local damage model.

The last zone to be better accounted for is that close to the impact point in which damage develops under highly confined conditions. This damage regime is only indirectly included in the elastoplastic description associated with the chosen HEL value, and is therefore not depicted in Figure 10 since only mode I cracks are explicitly described in the present model. However, it was shown that as a first approximation this type model is able to account for confined damage in rocks [41]. 


\section{Conclusion}

Edge-on-impact tests have been performed on sodalime silicate glass by using a soft bullet. These experiments allow for a visualization of the crack pattern by using a highspeed camera or a sarcophagus configuration. Fragmentation of impacted sodalime silicate glass is mainly caused by the growth of cracks nucleated on the surface of glass. Far from the impact surface, propagation of long cracks is always observed.

Numerically, the zone of multiple fragmentation is reproduced with the help of an anisotropic damage model based on a probabilistic analysis of multiple crack activations. Since the population of initiation sites is the key parameter that governs the cracking pattern, Weibull parameters representative of the surface and the bulk properties have been used. However, this model is not sufficient to account for the propagation of long cracks. An extension was therefore proposed.

As the propagation of long cracks is a non-local phenomenon for classical FE simulations, the zone obscured by one macrocrack is used to follow its propagation. This is achieved through a "meso-damage" model coupled with the previous one. 3D simulations prove that the different zones observed in edge-on-impact tests where mode I cracks initiate and propagate are reproduced by the present approach. A local approach would only give the initiation sites, and would not be able to capture the mesocrack formation. The different regimes of damage front velocities observed experimentally are then well captured in the simulations with the "meso-damage" model.

Even though the model proposed herein was used to simulate the dynamic fragmentation of glass, its domain of application may not be restricted to glass. The microscopic damage model was successfully used to analyze the fragmentation of SiC ceramics [29, $22,38,42]$, high performance concrete [43], and limestones [41]. In the two last cases, 
there were situations for which the crack length was greater than the element size, thereby calling for the same type of extensions as those proposed herein.

\section{Acknowledgements}

This work has been supported by a Saint-Gobain grant. 


\section{References}

1. J. Cagnoux, Déformation et ruine d'un verre pyrex soumis à un choc intense : étude expérimentale et modélisation du comportement, (Thèse d'Etat, University of Poitiers, 1985).

2. P. C. den Reijer, Impact on Ceramic Faced Armor, (PhD dissertation, Delft Technical University, 1991).

3. U. Hornemann, J. F. Kalthoff, H. Rothenhäusler, H. Senf and S. Winkler, Experimental Investigation of Wave and Fracture Propagation in Glass - Slabs Loaded by Steel Cylinders at High Impact Velocities, EMI report E 4/84, Weil am Rhein (Germany), 1984).

4. X. Brajer, F. Hild, S. Roux and R. Gy, Behavior of soda-lime glasses impacted by a soft bullet: experimental and numerical investigations, Proceedings 14th Dymat Technical Meeting, (EURODYMAT, 2002), 35-44.

5. X. Brajer, P. Forquin, R. Gy and F. Hild, The role of surface and volume defects in the fracture of glass under quasi-static and dynamic loadings, J. Non Cryst. Solids 316 (2003) 42-53.

6. E. Strassburger, P. Patel, J. W. McCauley, C. Kovalchick, K. T. Ramesh and D. W. Templeton, High-speed transmission shadowgraphic and dynamic photoelasticity study of stress wave and impact damage propagation in transparent materials and laminates using the edge-on impact method, Proceedings 23rd international symposium on ballistics, 2007.

7. C. Denoual, C. E. Cottenot and F. Hild, Analysis of the Degradation Mechanisms in an Impacted Ceramic, in: S. C. Schmidt, D. P. Dandekar and J. W. Forbes, eds., Proceedings Shock Compression of Condensed Matter, (AIP Press, New York (USA), 1998), 427-430.

8. K. Ravi-Chandar, Dynamic fracture of nominally brittle materials, Int. J. Fract. 90 (1998) 83-102.

9. X.-P. Xu and A. Needleman, Numerical simulations of fast crack growth in brittle solids, J. Mech. Phys. Solids 42 (1994) 1397-1434.

10. G. T. Camacho and M. Ortiz, Computational Modelling of Impact Damage in Brittle Materials, Int. J. Solids Struct. 33 [20-22] (1996) 2899-2938.

11. S. Mastilovic and D. Krajcinovic, High-Velocity Expansion of a Cavity within a Brittle Material, J. Mech. Phys. Solids 47 (1999) 577-600. 
12. F. Zhou and J.-F. Molinari, Stochastic fracture of ceramics under dynamic tensile loading, Int. J. Solids Struct. 41 (2004) 65736596.

13. J. J. Monaghan, Smoothed Particle Hydrodynamics, Annu. Rev. Astron. Astrophys. 30 (1992) 543-574.

14. L. D. Libersky and A. G. Petscheck, High Strain Lagrangian Hydrodynamics, J. Comp. Phys. 109 (1993) 67-75.

15. S. Hiermaier and W. Riedel, Numerical Simulation of Failure in Brittle Materials using Smooth Particle Hydrodynamics, Proceedings New Models and Numerical codes for Schock Wave Processes in Condensed Media, 1997.

16. G. R. Johnson and T. J. Holmquist, An Improved Computational Constitutive Model for Brittle Materials, High Pres. Sci. Tech. 2 (1992) 981-984.

17. B. Budiansky and R. J. O'Connell, Elastic Moduli of a Cracked System, Int. J. Solids Struct. 12 (1976) 81-97.

18. L. G. Margolin, Elasticity Moduli of a Cracked Body, Int. J. Fract. 22 (1983) 65-79.

19. L. M. Taylor, E.-P. Chen and J. S. Kuszmaul, Microcrack-Induced Damage Accumulation in brittle Rocks under Dynamic Loading, Comp. Meth. Appl. Mech. Eng. 55 (1986) 301320.

20. A. M. Rajendran, Modeling the Impact Behavior of AD85 Ceramic under Multiaxial Loading, Int. J. Impact Eng. 15 [6] (1994) 749-768.

21. C. Denoual, C. E. Cottenot and F. Hild, On the Identification of Damage during Impact of a Ceramic by a Hard Projectile, Proceedings 16th International Conference on BALLISTICS, (APDS, Arlington (USA), 1996), 541-550.

22. C. Denoual and F. Hild, Dynamic Fragmentation of Brittle Solids: A Multi-Scale Model, Eur. J. Mech. A/Solids 21 [1] (2002) 105-120.

23. H. D. Espinosa, P. D. Zavattieri and S. K. Dwivedi, A Finite Deformation Continuum/Discrete Model for the Description of Fragmentation and Damage in Brittle Materials, J. Mech. Phys. Solids 46 (1998) 1909-1942.

24. EN1063 standard, Glass in building Security glazing Testing and classification of resistance against bullet attack, CEN European Committee for Standardization (1999).

25. K. F. Graff, Wave Motion in Elastic Solids, (Clarendon Press, Oxford (UK), 1975). 
26. R. Gulino and S. L. Phoenix, Weibull Strength Statistics for Graphite Fibres Measured from the Break Progression in a Model Graphite/Glass/Epoxy Microcomposite, J. Mater. Sci. 26 [11] (1991) 3107-3118.

27. D. Jeulin, Modèles morphologiques de structures aléatoires et changement d'échelle, (thèse d'Etat, University of Caen, 1991).

28. K. Ravi-Chandar and B. Yang, On the role of microcracks in the dynamic fracture of brittle materials, J. Mech. Phys. Solids 45 [4] (1997) 535-563.

29. C. Denoual and F. Hild, A Damage Model for the Dynamic Fragmentation of Brittle Solids, Comp. Meth. Appl. Mech. Eng. 183 (2000) 247-258.

30. A. M. Freudenthal, Statistical Approach to Brittle Fracture, in: Fracture, H. Liebowitz, eds., (Academic Press, New York (USA), 1968), 591-619.

31. D. G. S. Davies, The Statistical Approach to Engineering Design in Ceramics, Proc. Brit. Ceram. Soc. 22 (1973) 429-452.

32. D. R. Oakley, An Empirical Study of the Effect of Stressed Area on the Strength of Float Glass Surfaces, J. Non-Cryst. Solids 196 (1996) 134-138.

33. R. Gy and C. Guillemet, Characterization of a mode of rupture of glass at $610^{\circ} \mathrm{C}$, in: The Physics of Non-Crystalline Solids, L. D. Pye, W. C. L. Course and H. J. Stevens, eds., (Taylor and Francis, London (UK), 1992.

34. W. Weibull, A Statistical Theory of the Strength of Materials, (Roy. Swed. Inst. Eng. Res., Report 151, 1939).

35. Y. N. Rabotnov, Creep Problems in Structural Members, (North-Holland, Amsterdam (the Netherlands), 1969).

36. D. E. Grady and M. E. Kipp, Continuum Modeling of Explosive Fracture in Oil Shale, Int. J. Rock Min. Sci. \& Geomech. Abstr. 17 (1980) 147-157.

37. A. Brara and J. Klepaczko, An Experimental Method for Dynamic Tensile Testing of Concrete by Spalling, Int. J. Impact Eng. 25 (2001) 387-409.

38. F. Hild, X. Brajer, C. Denoual and P. Forquin, On the Probabilistic-Deterministic Transition Involved in a Fragmentation Process of Brittle Materials, Comput. Struct. 81 [12] (2003) 1241-1253.

39. E. M. Pugh, R. v. Heine-Geldern, S. Foner and E. C. Mutschler, Glass Cracking Caused by High Explosives, J. Appl. Phys. 23 (1952) 48-53. 
40. J. Lemaitre, A Course on Damage Mechanics, (Springer-Verlag, Berlin (Germany), 1992).

41. S. Grange, P. Forquin, S. Mencacci and F. Hild, On the Dynamic Fragmentation of two Limestones using Edge-on Impact Tests, Int. J. Impact Eng. 35 [9] (2008) 977-991.

42. P. Forquin, L. Tran, P.-F. Louvigné, L. Rota and F. Hild, Effect of Aluminum Reinforcement on the Dynamic Fragmentation of SiC Ceramics, Int. J. Impact Eng. 28 (2003) 1061-1076

43. P. Forquin and F. Hild, Dynamic Fragmentation of an Ultra-High Strength Concrete during Edge-On Impact Tests, ASCE J. Eng. Mech. 134 [4] (2008) 302-315. 


\section{List of Figures}

1 Sequence of pictures of the impact of a BR4 bullet (velocity $=430 \mathrm{~m} / \mathrm{s}$ ) on a $100 \times 100 \times 8 \mathrm{~mm}^{3}$ glass target. The dark areas correspond to damaged zones. The dashed line shows the main crack used in Figure 9 for comparison between predicted and experimental results. . . . . . . . 25

2 Cracking pattern of impacted glass (44 Magnum bullet, $V=330 \mathrm{~m} / \mathrm{s}$ ) obtained with a sarcophagus configuration. Near the impact zone, the material is fully comminuted. Far from impact, long cracks are observed. 26

3 Examination domains in tension. The dots depict points that are potential initiation sites. Left) The tensile stress rate is low; one single crack has initiated, leading to the entire obscuration of the element. Right) The tensile stress rate is high; there is a competition between the stress increase and the obscuration of the element. Several cracks are initiated.

4 Average ultimate tensile strength vs. strain rate for a $10 \mathrm{~mm}^{3}$ element made of sodalime silicate glass (the surface is endowed with Weibull parameters $m_{s}=7, \sigma_{0 s}=100 \mathrm{MPa}, S_{\text {eff }}=100 \mathrm{~cm}^{2}$ [32], whereas the bulk is characterized by $m_{v}=30, \sigma_{0 v}=3 \mathrm{GPa}, V_{\mathrm{eff}}=10^{-6} \mathrm{~mm}^{3}[33]$ ).

5 FE simulations of one quarter of an EOI configuration. The damage variable $D_{1}$ associated with the first (maximum) principal stress shows the location of crack initiations. . . . . . . . . . . . . 29

6 Schematic view of a propagation of a long crack via the meso-damage model. The first step (left) corresponds to distributed damage modeled by damage variables $D_{i}$. The second step (right) corresponds to coalescence in one element described by meso-damage variables $\Delta_{i}$. . . . . . 30 
7 Comparison of the space-time diagrams of the damage variable $\Delta_{1}$ for two different meshes: 4 elements per mm (a) and 16 elements per mm (b). The white zone corresponds to $\Delta_{1}$ greater than $0.85 . \ldots 31$

8 Contact force vs. time for an impact of a BR4 bullet on a sodalime silicate target determined by a 3D FE simulation. . . . . . . . . . . . 32

9 Space-time diagram of the damage variable $\Delta_{1}$ obtained with the mesodamage model. The white zone corresponds to $\Delta_{1}$ greater than 0.85 . It is compared with an experimental crack front velocity evaluated from Figure 1 (dotted line). . . . . . . . . . . . . . . . 33

10 FE simulations of one quarter of an EOI configuration. Top: the damage variable $\Delta_{1}$ associated with the first (maximum) principal stress shows the location of cracks. Bottom: the damage variable $D_{1}$ associated with the first principal stress shows the location of crack initiations. . . . . . 34 

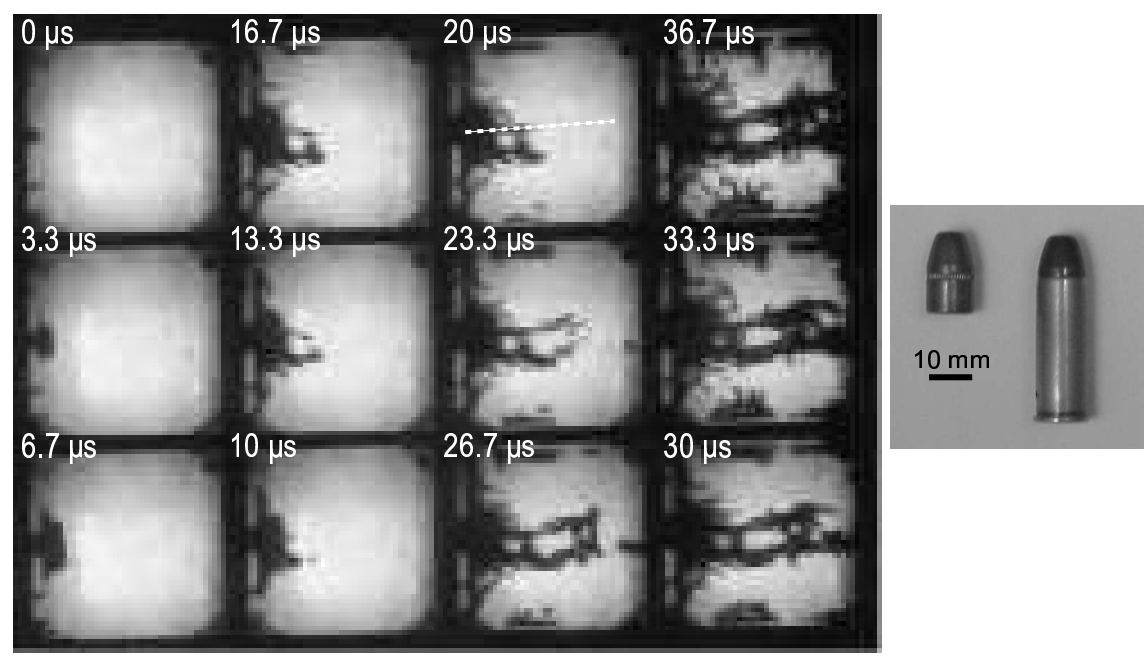

$-20 \mathrm{~mm}$

Fig. 1 Sequence of pictures of the impact of a BR4 bullet (velocity $=430 \mathrm{~m} / \mathrm{s}$ ) on a $100 \times$ $100 \times 8 \mathrm{~mm}^{3}$ glass target. The dark areas correspond to damaged zones. The dashed line shows the main crack used in Figure 9 for comparison between predicted and experimental results. 


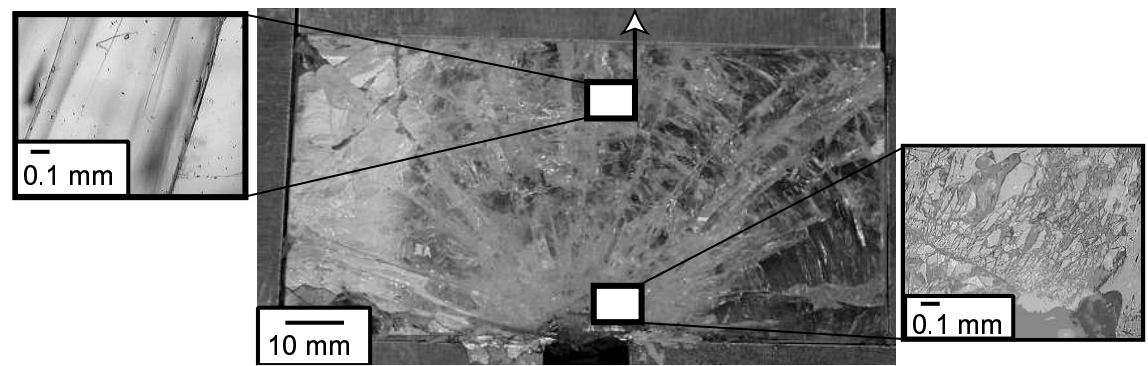

Fig. 2 Cracking pattern of impacted glass (44 Magnum bullet, $V=330 \mathrm{~m} / \mathrm{s}$ ) obtained with a sarcophagus configuration. Near the impact zone, the material is fully comminuted. Far from impact, long cracks are observed. 

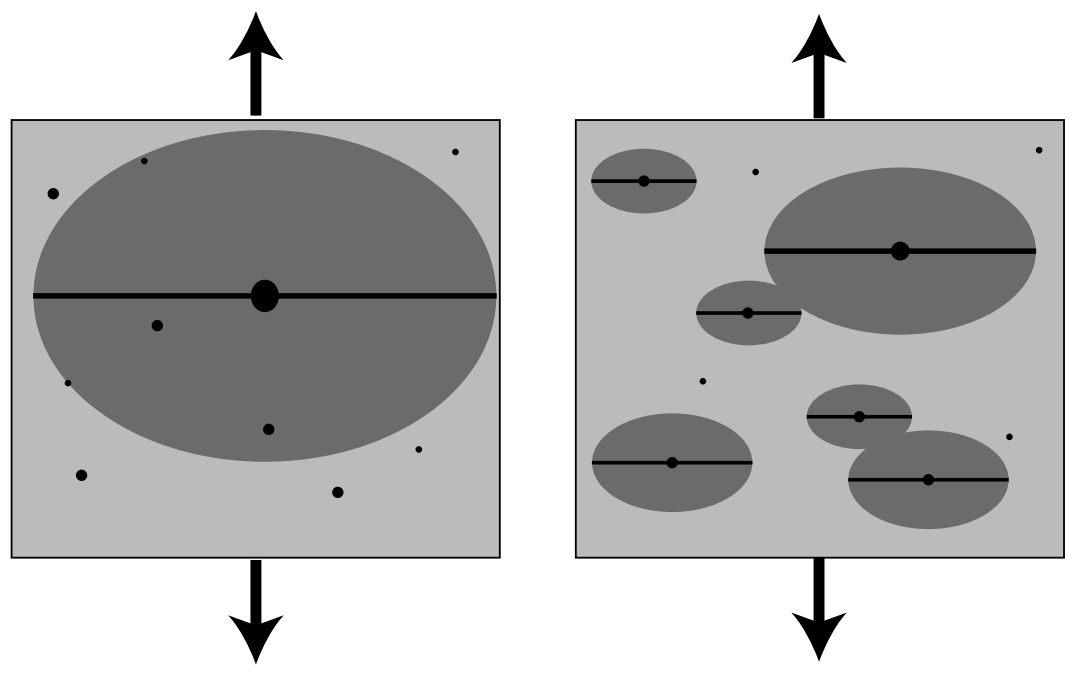

Fig. 3 Examination domains in tension. The dots depict points that are potential initiation sites. Left) The tensile stress rate is low; one single crack has initiated, leading to the entire obscuration of the element. Right) The tensile stress rate is high; there is a competition between the stress increase and the obscuration of the element. Several cracks are initiated. 


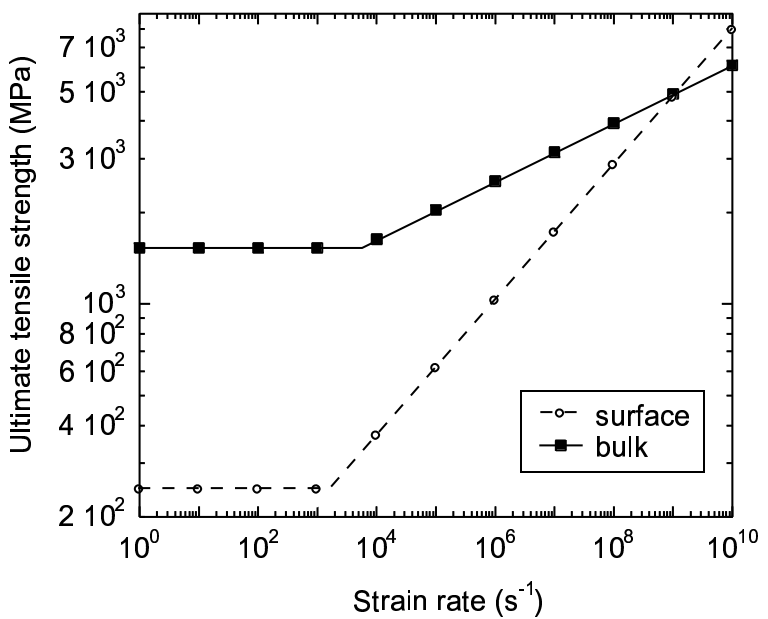

Fig. 4 Average ultimate tensile strength vs. strain rate for a $10 \mathrm{~mm}^{3}$ element made of sodalime silicate glass (the surface is endowed with Weibull parameters $m_{s}=7, \sigma_{0 s}=100 \mathrm{MPa}$, $S_{\text {eff }}=100 \mathrm{~cm}^{2}[32]$, whereas the bulk is characterized by $m_{v}=30, \sigma_{0 v}=3 \mathrm{GPa}, V_{\text {eff }}=$ $\left.10^{-6} \mathrm{~mm}^{3}[33]\right)$ 


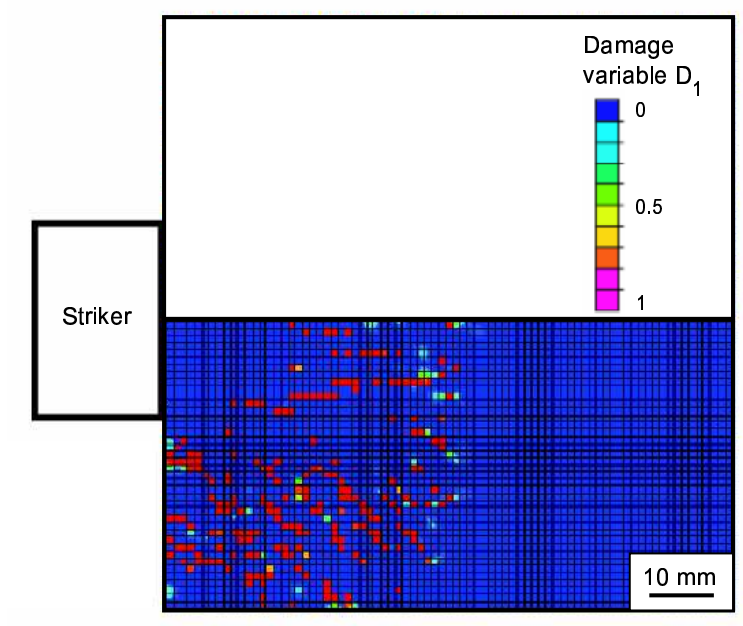

Fig. $5 \mathrm{FE}$ simulations of one quarter of an EOI configuration. The damage variable $D_{1}$ associated with the first (maximum) principal stress shows the location of crack initiations. 

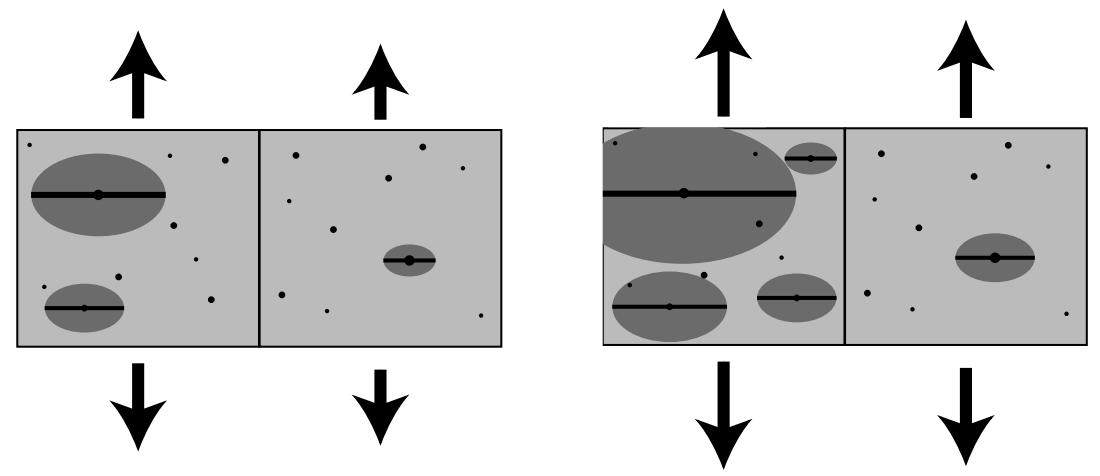

Fig. 6 Schematic view of a propagation of a long crack via the meso-damage model. The first step (left) corresponds to distributed damage modeled by damage variables $D_{i}$. The second step (right) corresponds to coalescence in one element described by meso-damage variables $\Delta_{i}$. 


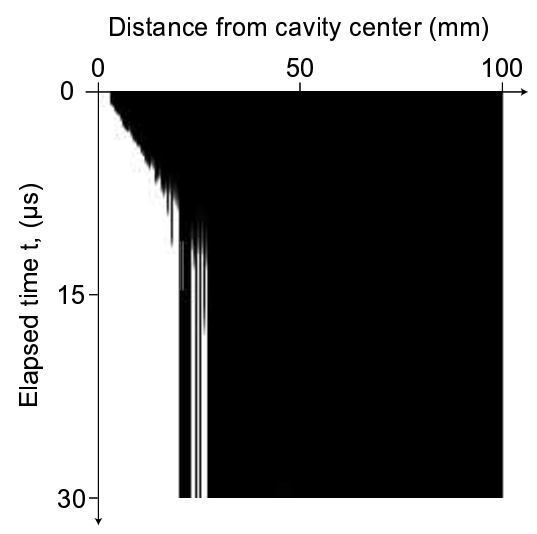

$-a-$

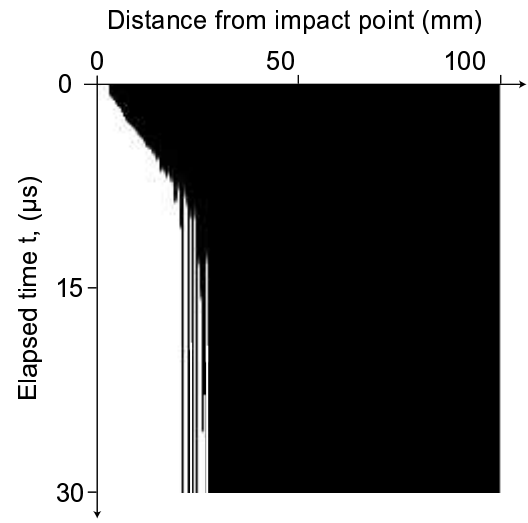

-b-

Fig. 7 Comparison of the space-time diagrams of the damage variable $\Delta_{1}$ for two different meshes: 4 elements per $\mathrm{mm}$ (a) and 16 elements per $\mathrm{mm}$ (b). The white zone corresponds to $\Delta_{1}$ greater than 0.85 


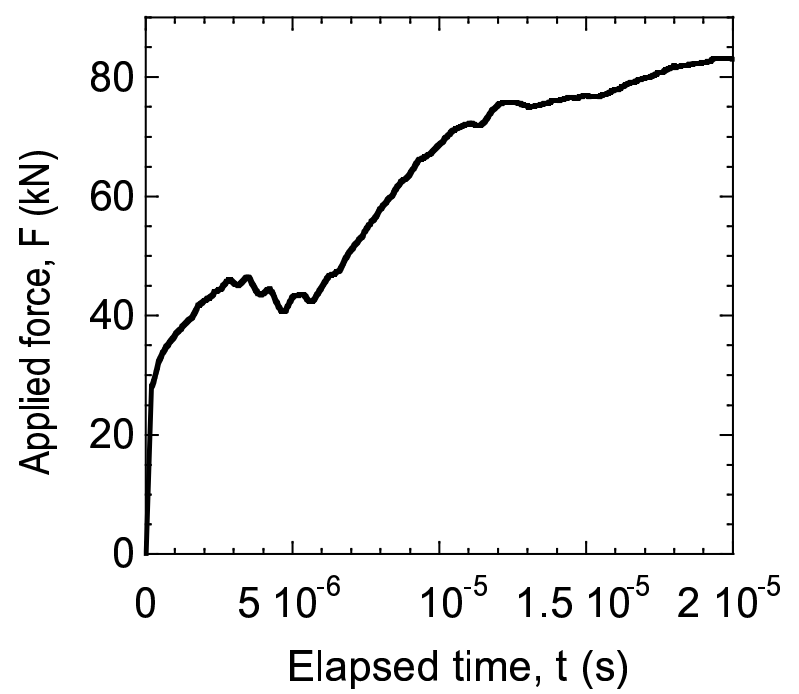

Fig. 8 Contact force vs. time for an impact of a BR4 bullet on a sodalime silicate target determined by a 3D FE simulation. 
Distance from impact point $(\mathrm{mm})$

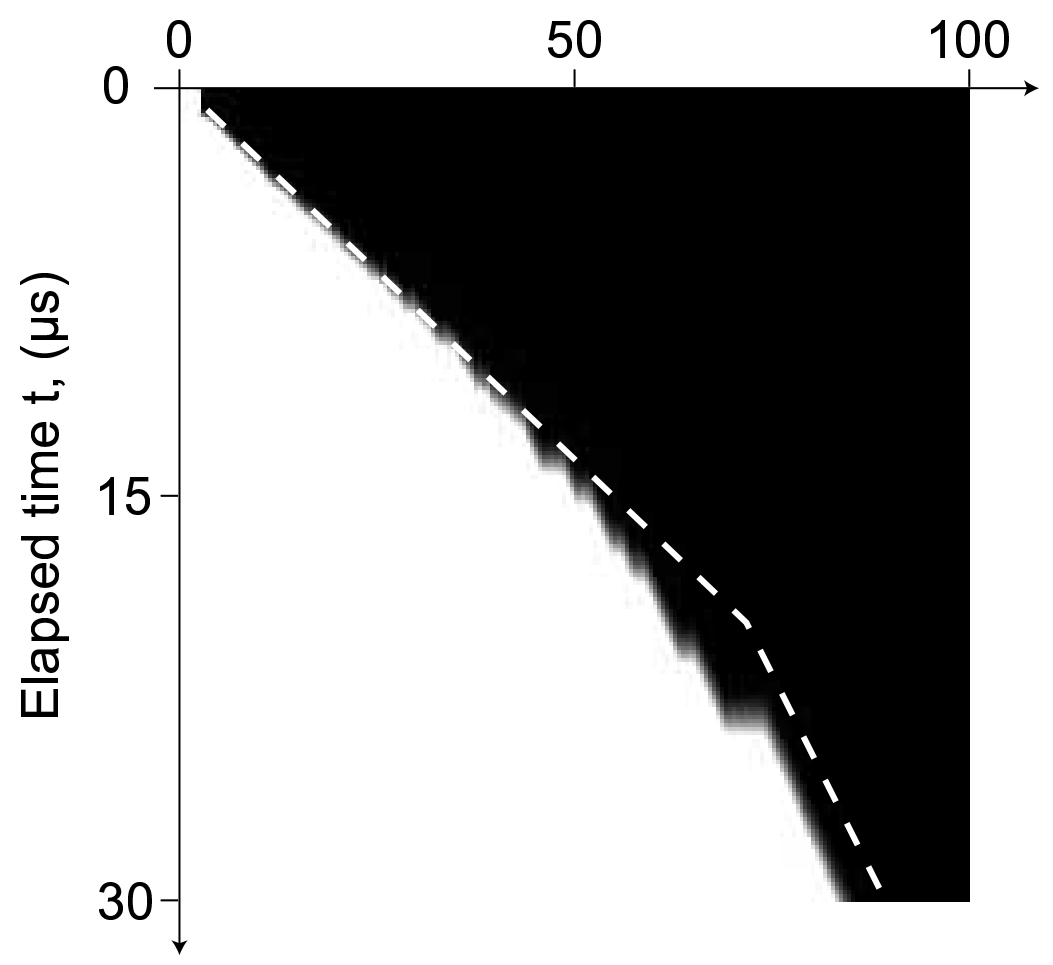

Fig. 9 Space-time diagram of the damage variable $\Delta_{1}$ obtained with the meso-damage model. The white zone corresponds to $\Delta_{1}$ greater than 0.85 . It is compared with an experimental crack front velocity evaluated from Figure 1 (dotted line). 


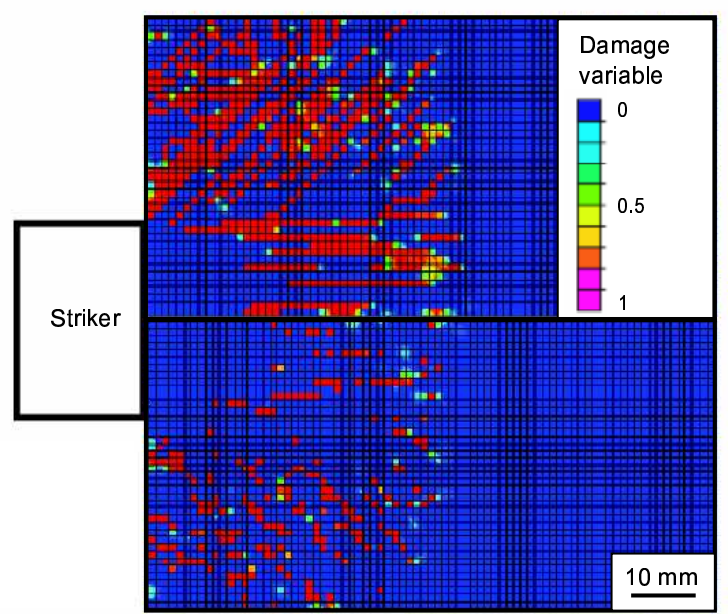

Fig. $10 \mathrm{FE}$ simulations of one quarter of an EOI configuration. Top: the damage variable $\Delta_{1}$ associated with the first (maximum) principal stress shows the location of cracks. Bottom: the damage variable $D_{1}$ associated with the first principal stress shows the location of crack initiations. 